\title{
Trapped in the net
}

\section{F. M. Riegler}

Dear Readers,

Today it is really hard to keep up with the continuous flow of novel information leaving us in a state of frustration and the feeling not being able to do a good and adequate job. Remains to be questioned if the present time requires a novel definition of our scopes and aims as modern physicians and surgeons?

Novel technologies are great and profoundly contribute to let us be better at our jobs. Surgery became hyper-minimal invasive, major procedures are conducted via mini single incisions. Optical devices allow fascinating magnified visualization of body cavities. The diagnostic tools became rapid and highly effective computer-based "play stations" allowing amazing visions into the complex texture of tissues, organs and pathophysiological processes. Medical science accelerates the conduct of knowledge acquisition. What is the price?

In addition to do our job as physicians and surgeons, we are forced to keep up with this race of knowledge and science. Otherwise it seems that we are thrown out of the game. It seems there is no quiet place out there for rest and stop. Going in line with this trend we are continuously pushed to assess and acquire new information. Thus computers, cell phones, laptops and iPads (+electronic radiations generated by the novel communication processes) place us within a continuous wave of global conduct. We became trapped in the net, so what?

Running and hunting is good, but not always. Consequently one will get exhausted and name a "six feet at last"-stone. Therefore, we have to redefine ourselves in this global competition and see if $W E$ really want to be part of it $A L L$ the TIME.

"Chill out" is important for the recreation and redefinition of our wants and needs. Otherwise we will directly drive into the burn out scenario and loss of life quality, happiness, well being. Man will cease like a fire in the wind, unfocused energy echoes along as part of the background noise.

The highly motivating scope is to combine efficient acquisition of knowledge, sound performance of patient care and relaxed recreation of our body, soul and mind. This is the basis for a balanced orchestration of modern life: take your time to keep up with the world and with

Correspondence: Franz Martin Riegler, M.D., Associate Professor of Surgery, Manometry Lab \& Department of Surgery, Medical University of Vienna, Waehringer Guertel 18-20, 1090 Vienna, Austria.

Fax: ++43-1-40400 3478

E-mail: Franz.Riegler@meduniwien.ac.at yourself. Let your life be a mix, as it is with music. Play the blues if you want blues, rock if you want to rock, jam if you want to jam, but do never forget to be $Y O U$. Expose yourself to good energies, communicate with persons with good vibrations. Distribute good energies and you will find yourself in a well-tuned groove. For surgeons it is important not to overestimate your performance, do not jump into the water, if you cannot swim, take your time to balance, step by step.

Going in line with the technological developments regarding the diagnosis and management of benign and malignant diseases we became highly effective in the treatment of pathologies limited to specific organs (i.e. liver, kidney, heart, lungs, gut, thyroids, and brain). However, this technology-driven specialization evolves at the

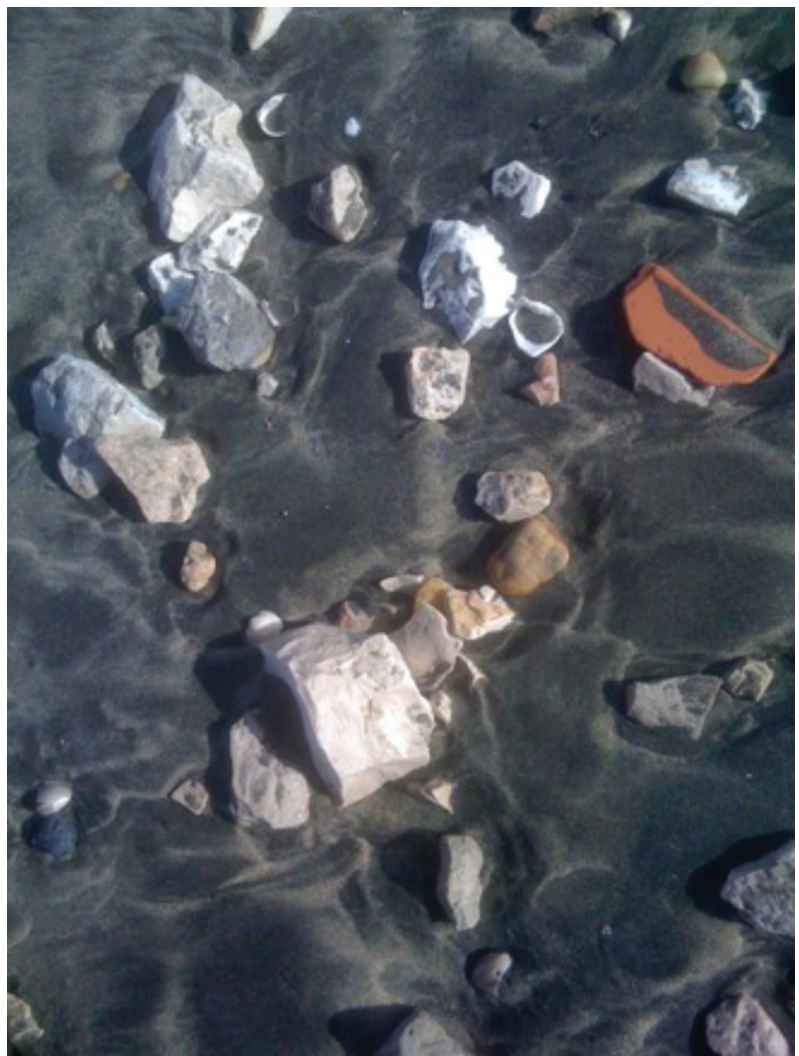

Fig. 1: Focused vision towards tissue texture. The image echoes that the modern diagnostic technologies drive attention towards the detail at the cost of universality. Image taken at a beach in Nettuno, Italy, Europe (iPhone and Mac Book Air technology) 


\section{Editorial}

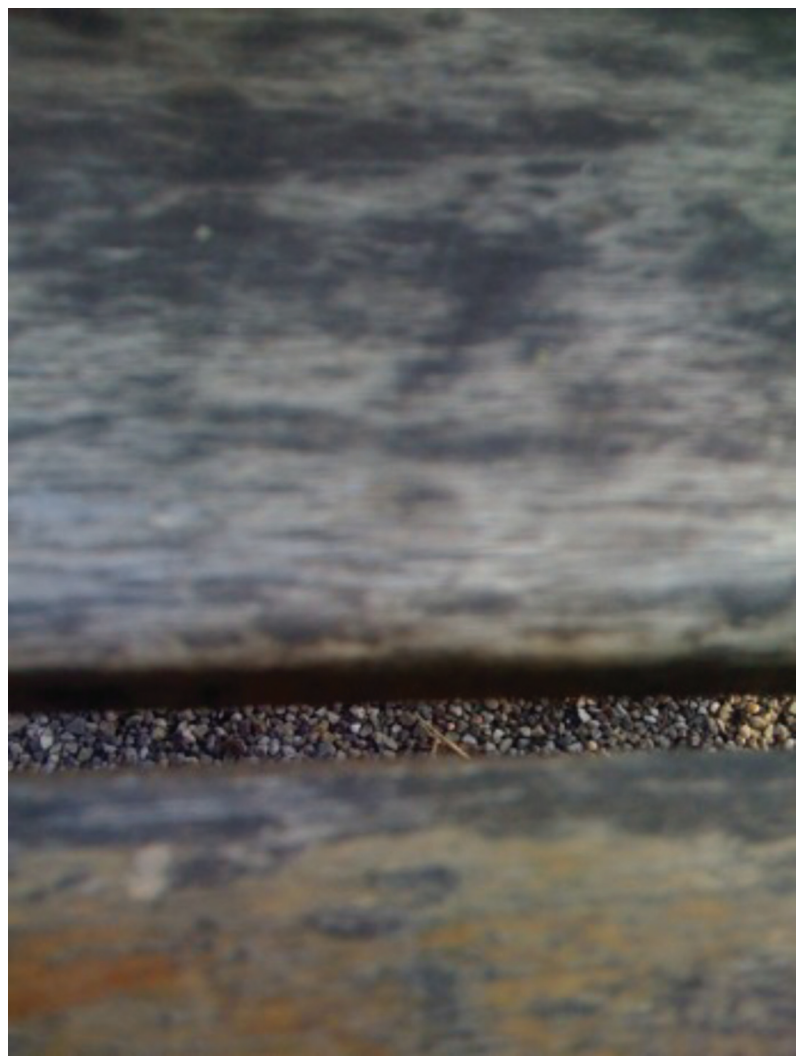

Fig. 2: The slot gives vision towards a granular tissue texture. The image refers to the idea of the author that technology-driven improvements of focus towards the detail occur at the cost and loss of the general view. The approach harbors the risk to miss the general view and changes that are out of focus (iPhone and Mac Book Air technology)

cost of a holistic approach, frequently leaving those unsatisfied with systemic unclear diseases (i.e. metabolic and chronic infections) (Figs. 1 and 2). Here we require physicians orchestrating the art of wide interdisciplinary differential diagnosis.

Let life be pictured as a mountain. Experience evolves with practice, you do not remember every single step that you took before reaching the top of a mountain, what remains is the wide vision, this bliss upon bliss state of the mood while watching the world around you, this is what remains (Fig. 3). Life is a continuous process, energy is absolute, positives and negatives arise due to individual

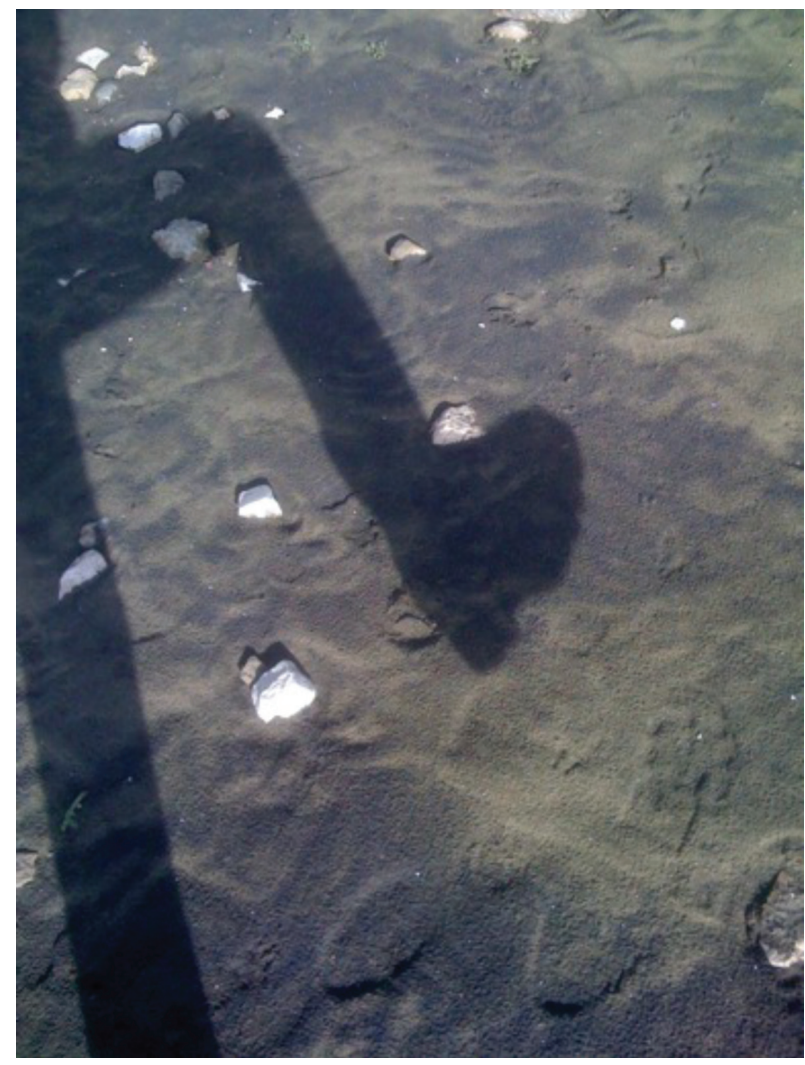

Fig. 3: We do not remember every step that we take to reach the mountain top. At the end remains the overview and the wide vision. This idea pictures the continuous process of stepping forward to reach a state of wide knowledge (wisdom), as outlined in the text (iPhone and Mac Book Air technology)

interpretation. Since you are the center of your world you have to define your aims and scopes. Cheer up in positivity, define the negatives and leave them out. Thus every day becomes an exciting positive challenge: follow the "riddim" of your pulse.

Franz Martin Riegler Editor in Chief

\section{Conflict of interest}

The author declares that there exists no conflict of interest. 